\title{
Unconventional topological phase transitions in helical Shiba chains
}

\author{
Falko Pientka, ${ }^{1}$ Leonid I. Glazman, ${ }^{2}$ and Felix von Oppen ${ }^{1}$ \\ ${ }^{1}$ Dahlem Center for Complex Quantum Systems and Fachbereich Physik, Freie Universität Berlin, 14195 Berlin, Germany \\ ${ }^{2}$ Department of Physics, Yale University, New Haven, Connecticut 06520, USA
}

(Received 13 January 2014; published 20 May 2014)

\begin{abstract}
Chains of magnetic impurities placed on a superconducting substrate and forming helical spin order provide a promising venue for realizing a topological superconducting phase. An effective tight-binding description of such helical Shiba chains involves long-range (power-law) hopping and pairing amplitudes which induce an unconventional topological critical point. At the critical point, we find exponentially localized Majorana bound states with a short localization length unrelated to a topological gap. Away from the critical point, this exponential decay develops a power-law tail. Our analytical results have encouraging implications for experiment.
\end{abstract}

Introduction. Currently, there is much excitement about topological superconducting phases [1,2]. There has been particular interest in one-dimensional topological superconducting phases with $p$-wave symmetry, engineered in hybrid systems based on conventional $s$-wave superconductors, and their Majorana end states [3-9]. A promising recent proposal involves a chain of magnetic impurities placed on an $s$-wave superconductor and corresponding experiments are under way [10-16] (see also [17-19]). It is envisioned that the magnetic impurities form a spin helix due to the Ruderman-KittelKasuya-Yosida (RKKY) interaction and induce Shiba bound states [20-23] in the superconducting host. These Shiba states hybridize to form bands, one each for the positive- and negative-energy Shiba states. Once the hybridization becomes strong enough for these two bands to overlap, the system can enter a topological superconducting phase.

The formation of Shiba bands can be modeled within a tight-binding Bogoliubov-de Gennes Hamiltonian [15]. The model Hamiltonian closely resembles Kitaev's toy model [3] of spinless $p$-wave superconductors, with the important distinction that the hopping and the pairing are long range [15]. In fact, Shiba states are known to exhibit a slow $1 / r$ decay away from the impurity for $r \ll \xi_{0}$ which crosses over into an exponential decay for $r \gg \xi_{0}$. For typical Shiba chains, the coherence length $\xi_{0}$ of the host superconductor is much larger than the impurity spacing $a$ which is comparable to the lattice spacing of the host superconductor. In numbers, one has $\xi_{0} / a \sim 10^{2}-10^{3}$ making a model with a pure $1 / r$ decay of hopping and pairing an excellent starting point. In the context of topological phases, this long-range coupling poses interesting questions. Most importantly, it is usually assumed [24] that the boundary modes of topological phases, such as Majorana end states, fall off exponentially into the bulk which seems incompatible with long-range coupling.

In this Rapid Communication, we provide an analytical theory for the surprising localization properties of the Majorana end states in helical Shiba chains, with important implications for experiment. A crucial property of helical Shiba chains is that as a consequence of long-range coupling, it displays an unconventional topological critical point as a function of the helix and Fermi wave vectors $k_{h}$ and $k_{F}$ [15]. The critical point is located exactly at $k_{h}=k_{F}$ in the limit $\xi_{0} \rightarrow \infty$ and remains close to it for finite $\xi_{0}$. Thus right at or near the critical point, the spin helix satisfies the condition for Bragg reflection which induces a strong tendency towards localizing the Majorana end states, competing with the delocalizing tendency of the long-range coupling. This may result in a localization length of the order of a few impurity sites, making isolated Majoranas accessible in experimentally feasible chains containing only a few dozen atoms.

Model. We consider a linear chain of magnetic impurities which are located at positions $x_{j}=j a$ and form a planar spin helix $\mathbf{S}_{j}$,

$$
\left(S_{j}\right)_{x}=S \cos 2 k_{h} x_{j}, \quad\left(S_{j}\right)_{y}=S \sin 2 k_{h} x_{j}, \quad\left(S_{j}\right)_{z}=0 .
$$

In the limit that the Shiba states of the individual impurities are deep, i.e., that their energy $\epsilon_{0}$ is close to the center of the gap, the system can be effectively described by a tight-binding Bogoliubov-de Gennes (BdG) Hamiltonian $\mathcal{H}=h \tau_{z}+\Delta \tau_{x}$ [15], where $\tau_{i}$ denotes Pauli matrices in particle-hole space, and $h$ as well as $\Delta$ are matrices in site space:

$$
h_{i j}=\epsilon_{0} \delta_{i j}-\Delta_{0}\left(1-\delta_{i j}\right) \frac{\sin k_{F} r_{i j}}{k_{F} r_{i j}} e^{-r_{i j} / \xi_{0}} \cos k_{h} x_{i j}
$$

and

$$
\Delta_{i j}=i \Delta_{0}\left(1-\delta_{i j}\right) \frac{\cos k_{F} r_{i j}}{k_{F} r_{i j}} e^{-r_{i j} / \xi_{0}} \sin k_{h} x_{i j} .
$$

Here, $\Delta_{0}$ denotes the pairing strength in the host superconductor and $r_{i j}=\left|x_{i j}\right|$ with $x_{i j}=x_{i}-x_{j}$. Note that both the hopping and pairing matrices are Hermitian, $h=h^{\dagger}$ and $\Delta=\Delta^{\dagger}$. Since the Shiba states are spin polarized, the pairing is effectively of $p$-wave nature, i.e., the pairing matrix is antisymmetric, $\Delta_{i j}=-\Delta_{j i}$. As explained above, this makes the Hamiltonian closely related to Kitaev's toy model [3] except for the long-range nature of the hopping and pairing amplitudes. In view of the large ratio $\xi_{0} / a$, we consider the limit $\xi_{0} \rightarrow \infty$ in the following unless otherwise stated.

This model has been discussed in detail in Ref. [15] and we briefly review its topological properties before deriving the unusual localization properties of the Majorana states near the Bragg point. Figure 1(a) reproduces the corresponding phase diagram as a function of Shiba bound state energy $\epsilon_{0}$ and Fermi wave vector $k_{F}$ [15]. The topological phase boundaries which appear in the phase diagram as diagonal lines occur when the chemical potential leaves the Shiba bands. These 

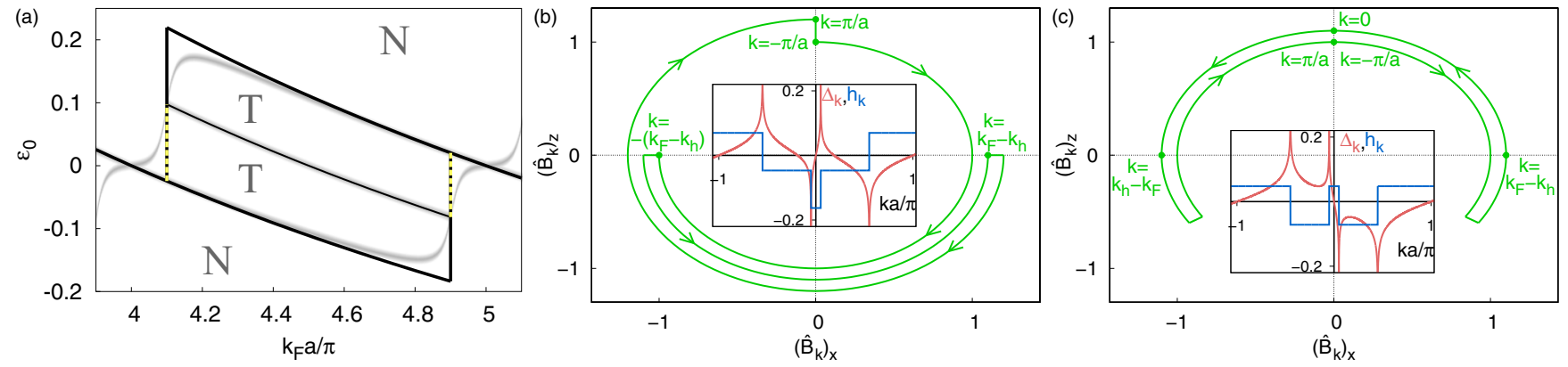

FIG. 1. (Color online) (a) Phase diagram for $k_{h}=0.1 \pi / a$ and $\xi_{0}=\infty$ (black phase boundaries) or $\xi_{0}=15 a$ (gray lines), with topological (T) and nontopological $(\mathrm{N})$ phases. (Energies are given in units of $\Delta_{0}$.) The yellow dashed line indicates the Bragg point $k_{F}=k_{h}$ with exponentially localized Majorana states $\left[|\beta|<1\right.$ in Eq. (6)]. For $\xi_{0}=\infty$, this coincides with the phase transition between the two- and single-channel phases. (b), (c) Winding of the unit vector $\hat{\mathbf{B}}_{k}=\mathbf{B}_{k} / B_{k}$ as $k$ is tuned across the Brillouin zone for (b) the single-channel and (c) the two-channel phase (partially shifted radially for visibility). While in the single-channel phase $\hat{\mathbf{B}}_{k}$ winds once around the origin; the winding is trivial in the two-channel phase, reflecting the topological phase transition. Insets: Dispersion $h_{k}$ and pairing $\Delta_{k}$ in the two phases. The two-channel dispersion has a second pair of Fermi points.

transitions are equivalent to those of the Kitaev chain and reflect a continuous gap closing and reopening. A different type of topological phase transition which emerges from the long-range coupling and has no analog in the Kitaev chain, occurs at the Bragg point $k_{h}=k_{F}$ (vertical lines) [25]. This is a discontinuous transition associated with the (dis)appearance of an additional pair of Fermi points near $k=0$ [see insets of Figs. 1(b) and 1(c)] modifying the system between effective single-channel and two-channel phases. In the nontopological two-channel phase, the cumulative hopping strength is finite across an even number of sites but vanishes across an odd number so that one can roughly think of the even and odd sites as two channels [26]. This happens for $k_{F}<k_{h}$, while even and odd sites are strongly coupled in the topological single-channel phase $k_{F}>k_{h}$. In some specific implementations, the RKKY interaction between the impurities is maximal at the wave vector $2 k_{F}$, so that the helix wave vector realizes the Bragg point $k_{h}=k_{F}[12,27,28]$. This would put the Shiba chain right at (for $\xi_{0} \rightarrow \infty$ ) or near (for large but finite $\xi_{0}$ ) an unconventional topological critical point.
For a planar spin helix, the Shiba chain obeys chiral symmetry, $\left\{\mathcal{H}, \tau_{y}\right\}=0$, which puts it in class BDI and in principle allows for a topological $\mathbb{Z}$ index [29]. To explore the discontinuous transition at the Bragg point more closely, we analyze the topological index of the two adjacent phases. To this end, we rewrite the Hamiltonian in momentum space, $\mathcal{H}_{k}=h_{k} \tau_{z}+\Delta_{k} \tau_{x}$, and determine the winding number of the two-component vector $\mathbf{B}_{k}=\left(\Delta_{k}, h_{k}\right)$ in the $x z$ plane as $k$ traverses the Brillouin zone from $-\pi / a$ to $\pi / a$ [see Figs. 1(b) and 1(c)]. This confirms the identification of the topologically trivial $\left(k_{F}<k_{h}\right)$ and nontrivial $\left(k_{F}>k_{h}\right)$ phases [30].

The transition between these phases at the Bragg point is reflected in the subgap states of long but finite chains. Their energies near $k_{F}=k_{h}$ are shown in Fig. 2(a). In the two-channel phase, one finds two subgap states for each end. These can be thought of as the hybridized Majorana states of the two channels. As $k_{F} \rightarrow k_{h}$, one subgap state merges with the quasiparticle continuum due to coupling with the opposite end of the chain, while the other approaches zero
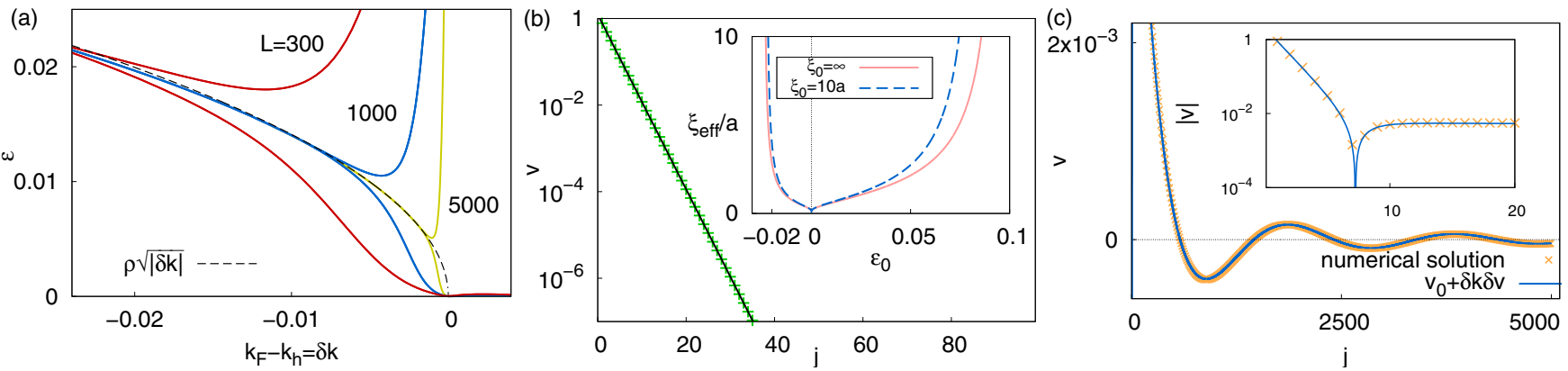

FIG. 2. (Color online) (a) Energy of the two positive-energy subgap states (in units of $\Delta_{0}$ ) in the nontopological two-channel phase $(\delta k<0)$ near the Bragg point, for $\xi_{0}=\infty$ and various chain lengths $L$. As $L \rightarrow \infty$, the two states become degenerate with energy $\sim \sqrt{|\delta k|}$ near the phase transition. At the critical point, one subgap state merges discontinuously with the quasiparticle continuum. For finite $L$, the discontinuity is smeared and the degeneracy is lifted on the scale $1 / L$. (b) Majorana wave function $v_{j}$ at the Bragg point $k_{F}=k_{h}$. The exact numerical solution of the BdG Hamiltonian (green crosses) agrees with the analytical solution (black line) in Eq. (6). Inset: Localization length $\xi_{\text {eff }}=a /$ ln $\left|\beta^{-1}\right|$ along the yellow line in the phase diagram in Fig. 1(a). The localization length is of order $a$ and decreases with increasing coherence length $\xi_{0}$. (c) Majorana wave function $v_{j}$ for $k_{F}=k_{h}+\delta k$ with $\delta k=0.003 / a$. The numerical solution of the BdG Hamiltonian (orange crosses) agrees with the analytical solution (blue line) as obtained by numerical evaluation of the inverse Laplace transform in Eq. (9). Inset: Blowup near the end of the chain emphasizing the initial exponential decay. Parameters: $\epsilon_{0}=0.03 \Delta_{0}, k_{h}=0.1 \pi / a$, and $k_{F}=4.1 \pi / a+\delta k$. 
energy and connects smoothly with the Majorana end state in the topological phase. Thus, right at the Bragg point, there is exactly one zero-energy state for each end of the chain. We now turn to an analytical theory of the Majorana bound state and the subgap spectrum both at and near the Bragg point.

Majorana bound state at the Bragg point $k_{F}=k_{h}$. We exploit the chiral symmetry of the Hamiltonian and rotate it into the Majorana basis in which $\mathcal{H}$ becomes purely off-diagonal in particle-hole space [31]. This is effected by a $\pi / 2$ rotation about the $x$ axis which transforms $\tau_{z} \rightarrow-\tau_{y}$ and keeps $\tau_{x}$ unchanged so that $\mathcal{H}=-h \tau_{y}+\Delta \tau_{x}$. Now, the equations for the zero-energy Majorana states with BdG spinor $(u, v)$ take the simple form

$$
u=0 \quad \text { and }(i h+\Delta) v=0
$$

for the Majorana localized at the left end of the chain, and $v=$ 0 and $(-i h+\Delta) u=0$ for the Majorana localized at the right end. (Note that this consistently neglects finite-size effects). Specifying to the left-end states for definiteness, one readily finds for $\mathcal{H}_{12}=i h+\Delta$, from Eqs. (2) and (3), that $\left(\mathcal{H}_{12}\right)_{j j}=$ $i \epsilon_{0}$ and

$$
\left(\mathcal{H}_{12}\right)_{i j}=-\frac{i \Delta}{k_{F} r_{i j}} e^{-r_{i j} / \xi_{0}} \sin \left(k_{F} r_{i j}-k_{h} x_{i j}\right)
$$

for $i \neq j$. Since $r_{i j}=\left|x_{i j}\right|$, the Bragg point $k_{h}=k_{F}$ has the remarkable property that $\mathcal{H}_{12}$ is a triangular matrix. This property immediately allows us to solve Eq. (4) by the ansatz $v_{j}=\beta^{j}$. Here, $j$ enumerates the sites starting with the left end of the chain. Indeed, with this ansatz, all components of the equation $\mathcal{H}_{12} v=0$ reduce to the same condition for $\beta$. Solving this condition, we find

$$
\beta=\frac{e^{a / \xi_{0}} \sin \left(k_{F} a \epsilon_{0} / \Delta\right)}{\sin \left[2 k_{F} a+k_{F} a \epsilon_{0} / \Delta\right]} .
$$

Obviously, this provides an exponentially localized Majorana solution as long as $|\beta|<1$. One can convince oneself that this condition is satisfied wherever the line $k_{F}=k_{h}$ is inside the topological phase for finite $\xi_{0}$. This region is marked by a yellow dashed line in Fig. 1(a). As shown in Fig. 2(b), this exact analytical result is in excellent agreement with numerical simulations.

This constitutes the central result of this work with remarkable implications: (i) Helical Shiba chains display an unconventional topological critical point at or in the immediate vicinity of the Bragg point $k_{F}=k_{h}$. (ii) At the Bragg point, they have Majonana end states which are exponentially localized even though the Hamiltonian allows for long-range hopping and pairing along the chain. (iii) The localization length $\xi_{\text {eff }}=a / \ln \left|\beta^{-1}\right|$ of the Majorana states is set by the spacing $a$ between the magnetic impurities and thus much shorter than the coherence length $\xi_{0}$ of the superconducting host. (iv) The Majorana end states at the Bragg point remain well defined and exponentially localized even in the limit $\xi_{0} \rightarrow \infty$ where the Bragg point coincides with the topological critical point. (v) We will see below that away from the Bragg point, the Majorana wave functions develop a power-law tail in addition to the initial exponential decay.

Physically, the strong localization for $k_{h}=k_{F}$ can be traced back to Bragg reflection. Similar to a Bragg mirror, the resonance between the oscillations of the Shiba states and the spin helix leads to destructive interference in one direction which neutralizes the long-range coupling. This explains that the localization length becomes of the order of the lattice spacing. More explicitly, the hopping and pairing terms are generically of the same order, but their relative magnitude depends sensitively on the wave vectors $k_{F}$ and $k_{h}$. At the resonance $k_{F}=k_{h}$, hopping and pairing between two arbitrary sites have equal magnitude but differ in parity. Hopping to left and right has the same sign and is thus even, whereas pairing is odd with opposite signs for the two directions.

Topological phase. When tuning away from the Bragg point, Bragg reflection is no longer perfect and the long-range character of the model is partially recovered. As a result, the wave function acquires a tail with a slow power-law decay as we will now show for the immediate vicinity of the Bragg point (in agreement with earlier numerical results [15]). Here we first focus on the topological phase $\left(k_{F}=k_{h}+\delta k\right.$ with $\delta k$ small and positive) and return to the nontopological phase $\left(k_{F}=k_{h}+\delta k\right.$ with $\delta k$ small and negative) further below.

For $\delta k$ small and positive, the matrices $\mathcal{H}_{12}$ and $\mathcal{H}_{21}$ are no longer triangular, but we still expect a localized Majorana state in a semi-infinite chain. We expand the eigenvalue problem to linear order in $\delta k$ and show that it reduces to an integral equation in a suitably taken limit when setting $\xi_{0} \rightarrow \infty$. The integral equation can then be solved by standard methods. To first order in $\delta k$, we rewrite $\mathcal{H}_{12} v=0$ as

$$
(M+\delta M)\left(v_{0}+\delta k \delta v\right)=0
$$

(see [26] for numerical support of this expansion). Here, $M$ is the upper triangular part of $\mathcal{H}_{12}$ with $M_{i i}=i \epsilon_{0}$, $M_{i j}=\left(-i \Delta / k_{F}\right) \sin \left(K r_{i j}\right) / r_{i j}$ for $i<j$ in terms of $K=$ $k_{F}+k_{h}$, and $\delta M$ is the lower triangular part with $\delta M_{i j}=$ $\left(-i \Delta / k_{F}\right) \sin \left(\delta k r_{i j}\right) / r_{i j}$ for $i>j$. To zeroth order in $\delta k$, we obtain $M v_{0}=0$ and thus $v_{0}$ coincides with the exponentially decaying solution at the Bragg point.

Next, we rewrite $\delta M$ as $\delta M_{i+j, i}=\left(-i \Delta / k_{F}\right) \delta k \sin y_{j} / y_{j}$ with $y_{j}=\delta k a j$. Thus $\delta M_{i j}$ varies only on large scales $r_{i j} \sim$ $1 / \delta k$ and we can take a continuum limit by considering $\delta k \rightarrow 0$ while keeping $y_{j}$ fixed. In this limit, $\delta M$ converges to a continuous matrix as a function of $y_{j} \rightarrow y$ and correspondingly, $\delta v$ should also have a well-defined continuum limit $\delta v \rightarrow \delta v(y)$ as a function of the scaled variable $y_{j}$. The existence of this continuum limit is readily confirmed by numerics and to linear order in $\delta k$, Eq. (7) yields an integral equation for $\delta v(y)$ [26],

$$
A \delta v(y)+\int_{0}^{y} d z \frac{\sin (y-z)}{y-z} \delta v(z)=-B \frac{\sin y}{y} .
$$

Here we defined $A=F\left(k_{F}+k_{h}\right)-\frac{k_{F} a \epsilon_{0}}{\Delta}, \quad B=$ $a \operatorname{sgn} \beta[(1+\beta) /(1-\beta)]^{1 / 2}, \quad$ and $\quad F(x)=\arctan \cot (x / 2)$. This integral equation can be solved in a standard manner by Laplace transform $\mathcal{L}$ which yields [26]

$$
\delta v(y)=-\mathcal{L}^{-1}\left[\frac{B \operatorname{arccot} s}{A+\operatorname{arccot} s}\right] \underset{y \rightarrow \infty}{\sim}-4 A B \frac{\sin y}{y \ln ^{2} y} .
$$

Corrections to the asymptote are suppressed by factors of $1 / \ln y$. Although our analytical analysis focuses on the vicinity of the Bragg point, the asymptotic decay is characteristic of the Majorana states in the entire topological phase when 
$\xi_{0}=\infty$ as previously established numerically [15]. Figure 2(c) shows that the analytical solution (9) is in excellent agreement with numerical results.

Nontopological phase. When the Bragg point $k_{F}=k_{h}$ falls into the topological phase for finite $\xi_{0}$ [i.e., along the yellow line in Fig. 1(a)], the nontopological side of the phase transition $(\delta k<0)$ can be understood as an effective two-channel wire [15]. Thus, this phase exhibits two subgap states for each end. In long chains, $L \rightarrow \infty$, their wave function and energy can be obtained analytically by an extension of the technique used for $\delta k>0$. Here, we sketch the results and defer details to the Supplemental Material [26].

The two positive-energy subgap states become degenerate for large $L$, and to leading order in $|\delta k|$ we find that their energy scales as $\epsilon \sim|\delta k|^{1 / 2}$, consistent with Fig. 2(a). Similarly, the Nambu wave function $(u, v)$ for the state at the left end [32] has $u_{i} \sim|\delta k|^{1 / 2} \exp (-\cot A|\delta k| a i)$ and $v \sim v_{0}+|\delta k| \delta v$, with $v_{0}$ the exponential solution at the critical point and $\delta v$ a power-law tail $\sim 1 / y \ln ^{2} y$ as for $\delta k>0$. The electron component $u$ decays exponentially with a decay length which diverges for $\delta k=0$, reflecting the phase transition and the disappearance of one subgap state. The hole component $v$ smoothly evolves into the Majorana bound state on the topological side.

Conclusions. As a consequence of the long-range coupling, helical Shiba chains display an unconventional topological critical point at the Bragg point $k_{F}=k_{h}$. We show that for finite chains, the Majorana end states persist at the critical point and display remarkable localization properties. By the competition between Bragg reflection and long-range coupling, the Majorana end states are exponentially localized at the critical point but develop a power-law tail in the topological phase. This contrasts in an interesting way with the decay of correlations around conventional critical points.

This is also an encouraging prediction for experiment, as the exponential localization at the critical point is on the scale of the lattice spacing and entirely unrelated to a topological gap. Thus, the Majorana end states may remain well localized even in chains whose length is comparable to the coherence length of the host superconductor. At the same time, the powerlaw localization within the topological phase raises interesting questions with regard to its stability against perturbations such as disorder.

Acknowledgments. We thank Ali Yazdani for stimulating discussions and Roman Lutchyn and Jelena Klinovaja for useful comments. We acknowledge financial support by the Helmholtz Virtual Institute "New states of matter and their excitations," SPP1285 of the Deutsche Forschungsgemeinschaft, the Studienstiftung d. dt. Volkes, and NSF DMR Grants No. 0906498 and No. 1206612. We are grateful to the Aspen Center for Physics, supported by NSF Grant No. PHYS106629 , for hospitality while this line of work was initiated.
[1] J. Alicea, Rep. Prog. Phys. 75, 076501 (2012).

[2] C. W. J. Beenakker, Annu. Rev. Condens. Matter Phys. 4, 113 (2013).

[3] A. Y. Kitaev, Phys. Usp. 44, 131 (2001).

[4] L. Fu and C. L. Kane, Phys. Rev. Lett. 100, 096407 (2008).

[5] R. M. Lutchyn, J. D. Sau, and S. Das Sarma, Phys. Rev. Lett. 105, 077001 (2010).

[6] Y. Oreg, G. Refael, and F. von Oppen, Phys. Rev. Lett. 105, 177002 (2010).

[7] J. Alicea, Y. Oreg, G. Refael, F. von Oppen, and M. P. A. Fisher, Nat. Phys. 7, 412 (2011).

[8] V. Mourik, K. Zuo, S. M. Frolov, S. R. Plissard, E. P. A. M. Bakkers, and L. P. Kouwenhoven, Science 336, 1003 (2012).

[9] A. Das, Y. Ronen, Y. Most, Y. Oreg, M. Heiblum, and H. Shtrikman, Nat. Phys. 8, 887 (2012).

[10] S. Nadj-Perge, I. K. Drozdov, B. A. Bernevig, and A. Yazdani, Phys. Rev. B 88, 020407(R) (2013).

[11] S. Nakosai, Y. Tanaka, and N. Nagaosa, Phys. Rev. B 88, 180503(R) (2013)

[12] J. Klinovaja, P. Stano, A. Yazdani, and D. Loss, Phys. Rev. Lett. 111, 186805 (2013).

[13] B. Braunecker and P. Simon, Phys. Rev. Lett. 111, 147202 (2013).

[14] M. M. Vazifeh and M. Franz, Phys. Rev. Lett. 111, 206802 (2013).

[15] F. Pientka, L. I. Glazman, and F. von Oppen, Phys. Rev. B 88, 155420 (2013).

[16] K. Pöyhönen, A. Westström, J. Röntynen, and T. Ojanen, Phys. Rev. B 89, 115109 (2014).

[17] T.-P. Choy, J. M. Edge, A. R. Akhmerov, and C. W. J. Beenakker, Phys. Rev. B 84, 195442 (2011).
[18] M. Kjaergaard, K. Wölms, and K. Flensberg, Phys. Rev. B 85, 020503 (2012).

[19] I. Martin and A. F. Morpurgo, Phys. Rev. B 85, 144505 (2012).

[20] L. Yu, Acta Phys. Sin. 21, 75 (1965).

[21] H. Shiba, Prog. Theor. Phys. 40, 435 (1968).

[22] A. I. Rusinov, Zh. Eksp. Teor. Fiz., Pis'ma Red. 9, 146 (1968) [JETP Lett. 9, 85 (1969)].

[23] A. V. Balatsky, I. Vekhter, and J.-X. Zhu, Rev. Mod. Phys. 78, 373 (2006).

[24] M. Z. Hasan and C. L. Kane, Rev. Mod. Phys. 82, 3045 (2010).

[25] Additional Bragg points exist for $2 k_{F}= \pm 2 k_{h}+2 \pi n$.

[26] See Supplemental Material at http://link.aps.org/supplemental/ 10.1103/PhysRevB.89.180505 where we discuss the twochannel phase and the case of finite $\xi_{0}$ and provide a detailed derivation of the Majorana wave function at and near the Bragg point.

[27] P. Simon, B. Braunecker, and D. Loss, Phys. Rev. B 77, 045108 (2008).

[28] B. Braunecker, P. Simon, and D. Loss, Phys. Rev. B 80, 165119 (2009)

[29] S. Ryu, A. P. Schnyder, A. Furusaki, and A. W. W. Ludwig, New J. Phys. 12, 065010 (2010).

[30] Incidentally, the two topological phases in Fig. 1(a) differ by the winding direction and have topological indices \pm 1 . Thus, the intermediate phase transition involves two simultaneous gap closings.

[31] I. Adagideli, P. M. Goldbart, A. Shnirman, and A. Yazdani, Phys. Rev. Lett. 83, 5571 (1999).

[32] The second positive-energy subgap state is localized at the right end with electron and hole components exchanged. The two negative-energy states are related to the positive ones by chiral symmetry. 